\title{
AERIAL POINT CLOUD CLASSIFICATION USING AN ALTERNATIVE APPROACH FOR THE DYNAMIC COMPUTATION OF K-NEAREST NEIGHBORS
}

\author{
Iuliana Maria Pârvu a *, E. Özdemir b, c, F. Remondino ${ }^{c}$ \\ a* National Center of Cartography, Cartography and Photogrammetry Department, 012101 / Doctoral School, Technical University \\ of Civil Engineering of Bucharest, 020396, Romania / e-mails: * iuliana.parvu@cngcft.ro / iuliana-maria.bina@ phd.utcb.ro \\ b 3D Optical Metrology (3DOM) unit, Bruno Kessler Foundation (FBK), Trento, Italy, e-mails: eozdemir@ fbk.eu, remondino@ @bk.eu \\ c Space Center, Skolkovo Institute of Technology (SKOLTECH), Moscow, Russia, e-mail: emre.ozdemir@skoltech.ru
}

Received: 30.05.2020 / Accepted: 10.07.2020/ Revised: 06.10.2020 / Available online: 15.12.020

DOI: 10.2478/jaes-2020-0023

KEY WORDS: point cloud, classification, region growing, eigenfeature, neighbourhood.

\begin{abstract}
:
The paper reports some methods to select the optimal number of neighbors and to use eigenfeatures for aerial point cloud classification. In the literature, the neighborhood selection is performed using different methods. In this paper, we propose an approach that uses the region growing algorithm. The input data is an aerial point cloud, part of the Romanian Dataset from LAKI II Project. To test our approach, we used a small dataset from the city of Marghita, Bihor County. We report the technical background for classification process and all technical details of the workflow used with insight analyses and comparisons. The work was realized within the VOLTA project (VOLTA, 2017), a RISE Marie-Curie action designed to do research and innovation activities among partners and to exchange knowledge, methods and workflows in the geospatial field.
\end{abstract}

\section{INTRODUCTION}

The light detection and ranging system (LiDAR) is a very common surveying technology for acquiring 3D spatial data. LiDAR systems capture data in 3D point cloud format and these data can be used to accurately localize objects and characterize their structure (Wen et al., 2020).

LiDAR systems are used in airborne, terrestrial, and mobile scanning, and provide detailed digital terrain models and record landscape in 3D. In the last twenty years, this technology has evolved significantly such that it is now possible to scan over 1 million points per second within $5 \mathrm{~mm}$ accuracy (Gaurav, 2017).

3D point cloud analysis has become an important task for mapping purposes as well as for infrastructure and environmental monitoring. The most well-known applications of 3D point clouds are topographic mapping, forest monitoring (Zhao et al., 2009), object detection (Serna and Marcotegui, 2014), 3D building reconstruction, and 3D city models generation (Ben-Joseph et al., 2001).

The classification aims at uniquely assigning a class label to each 3D point of a given 3D point cloud. Supervised classification relies on exploiting a set of training samples in order to train the classifier so that for unseen data it can predict the corresponding class. Point cloud classification allows categorizing points into different classes like bare-soil, buildings, vegetation, water, etc. Despite the evolution of available software, point cloud classification still remains a challenge and an active area of research.
The workflow of assigning a label to every point in the point cloud starts with the optimal neighborhood selection and continues with feature extraction and selection of the best suitable features. The final step is to perform the supervised classification and to assess the accuracy.

In this paper, we present an alternative approach for the classification of aerial point clouds in urban scenes, where we focus on a different workflow for computing the optimal number of neighbors, based on region growing algorithm. Starting with this approach, we have tested different combinations of eigenfeatures to see which better describes the point cloud. In the process of classification, we have used a basic approach for multi-class classification task, called One vs One machine learning classifier. The work was performed during a secondment of the first author in FBK (Trento, Italy) within the VOLTA project activities.

\section{RELATED WORKS}

A very straightforward definition for the process of classification is to transform unlabeled data into labeled data based on the feature extraction (Figure 1).

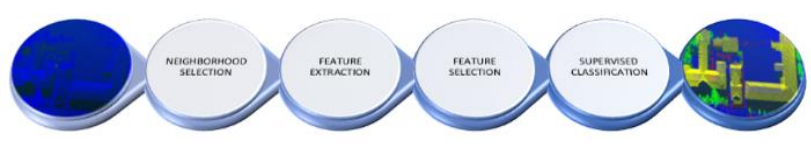

Figure 1. From unclassified to classified data

\footnotetext{
* Corresponding author: Iuliana Maria Pârvu, National Center of Cartography, Cartography and Photogrammetry Department, 012101 / Doctoral School, Technical University of Civil Engineering of Bucharest, 020396, Romania, e-mails: iuliana.parvu@cngcft.ro / iuliana-maria.bina@phd.utcb.ro.
} 
A feature describes a property by which we distinguish real or abstract elements or objects (Weinmann, 2013). So, depending on the application, some features may be suitable and some may not.

When analysing images, the visual features refer to colour, intensity, shape, size, orientation, or texture. Whereas those features may be rather global or local. The local features only rely on a small spatial image neighborhood, which is a regular raster.

With 3D point clouds, we may compute different feature types, like point attributes, shape, and local features (Weinmann, 2016). The features in this category are intensity, colour, spatial $3 \mathrm{D}$ coordinates or more abstract information like planarity, surface variation, etc.

\subsection{Eigenfeatures Extraction}

The Principal Component Analysis (PCA) is an orthogonal transformation technique used to convert a set of points with possibly correlated variables to another set of points with linearly uncorrelated variables called principal components, where the first principal component has the largest variance and every successive component is orthogonal to the preceding components (Jolliffe, 2002). The eigenvalues $\left(\lambda_{1}, \lambda_{2}, \lambda_{3}\right)$ contain valuable information for characterizing the local $3 \mathrm{D}$ shape at a considered 3D point. Based on these values, we can compute different eigenfeatures.

The eigenvalue-based features (Figure 2) are computed using the following formulas (Weinmann, 2016):

$$
\begin{array}{lll}
\text { - } & \text { linearity } & L=\frac{\lambda_{1}-\lambda_{2}}{\lambda_{1}} \\
\text { - } & \text { planarity } & P=\frac{\lambda_{2}-\lambda_{3}}{\lambda_{1}} \\
\text { - } & \text { scattering } & S=\frac{\lambda_{3}}{\lambda_{1}}
\end{array}
$$

- $\quad$ surface variation $C=\frac{\lambda_{1}}{\lambda_{1}+\lambda_{2}+\lambda_{3}}$

- omnivariance $\quad O=\sqrt[3]{\left(\lambda_{1} \lambda_{2} \lambda_{3}\right)}$

- $\quad$ anisotropy

$$
A=\frac{\lambda_{1}-\lambda_{3}}{\lambda_{1}}
$$

- eigenentropy $E=-\sum_{i=1}^{3} \lambda_{i} \ln \left(\lambda_{i}\right)$

- $\quad$ sum of eigenvalues $\sum=\lambda_{1}+\lambda_{2}+\lambda_{3}$
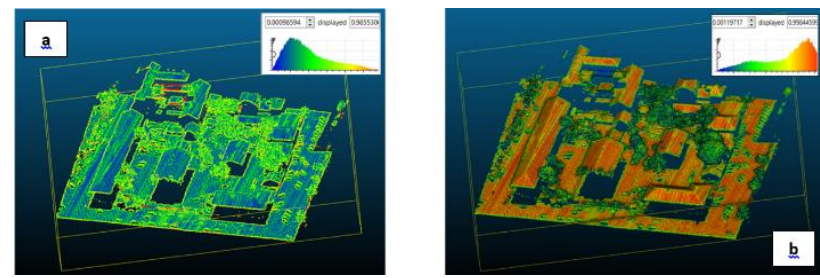
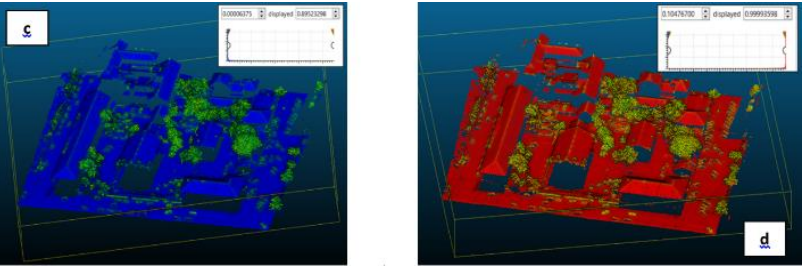

Figure 2. Examples of eigenvalue-based features (a. linearity, $b$. planarity, $c$. surface variation, $d$. anisotropy)

\subsection{Neighborhood Selection}

When working with $3 \mathrm{D}$ data, we have to select very well the neighborhood to compute the features. This step can be performed in many ways. The most common way is to consider the geometric size of the neighborhood (Figure 3) spherical, or cylindrical with a fixed radius (Lee and Schenk, 2002).

A spherical neighborhood around a 3D point is formed by all those $3 \mathrm{D}$ points within a sphere centered at the point and parametrised with a fixed radius. A cylindrical neighborhood around a $3 \mathrm{D}$ point is formed by all those $3 \mathrm{D}$ points whose $2 \mathrm{D}$ projections onto a plane are within a circle centred at the projection of the point and parameterized with a fixed radius (Filin and Pfeifer, 2005).
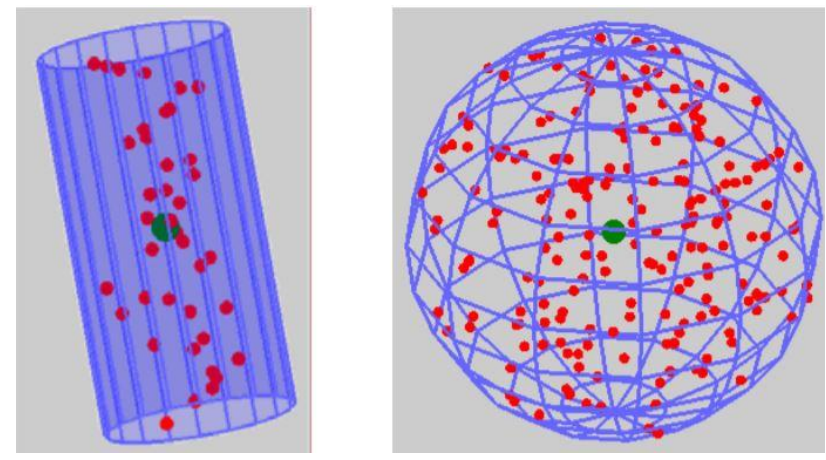

Figure 3. Geometric sized neighborhoods (Gross et al., 2007)

The 3D point cloud data may have a varying point density. This situation makes the geometric sized neighborhoods with a fixed radius for all points not suitable. A better choice is to use a flexible neighborhood given by the $\mathbf{k}$ closest neighbors in $3 \mathrm{D}$ space. Here, the problem is to determine the optimal value for $\mathbf{k}$. The first approach, called dimensionality-based scale selection (Figure 4), chooses the most appropriate scale of analysis among a range of potential values using three low-level geometrical shape features describing the dimensionality of the local point set (1D-2D-3D). Based on the eigenvalues, we compute the eigenfeatures that best describe the spaces: $L_{\lambda}$ linearity for $1 \mathrm{D}, P_{\lambda}$ planarity for $2 \mathrm{D}$, and $S_{\lambda}$ scattering for 3D (Demantké et al., 2011).

$$
E_{\text {dim }}=-L_{\lambda} \ln \left(L_{\lambda}\right)-P_{\lambda} \ln \left(P_{\lambda}\right)-S_{\lambda} \ln \left(S_{\lambda}\right)
$$

where: $E_{d i m}=$ eigenentropy.

The equations for the eigenfeatures - linearity, planarity, and scattering are given in formulas (1), (2) and (3). The lower the eigenentropy $E_{\operatorname{dim}}$ is the more one-dimensionality prevails over the other ones. This criterion permits determining the best radius for which the eigenentropy is minimal. 


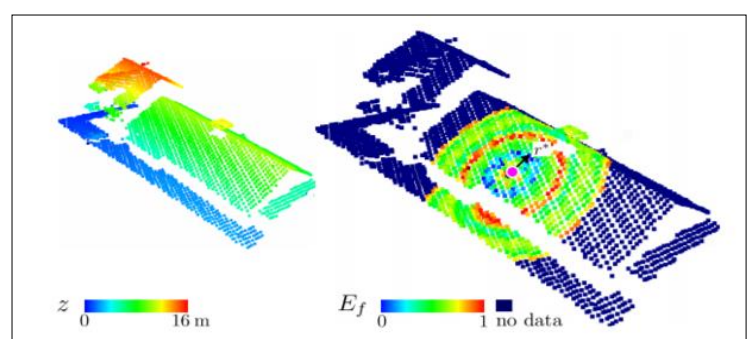

Figure 4. The relevance of the entropy feature for scale selection (Demantké et al., 2011)

A second well-known approach called eigenentropy-based scale selection is based on minimizing the measure of eigenentropy over varying scales to derive optimized features with higher distinctiveness in the step of feature extraction (Weinmann et al., 2014).

$$
E_{\lambda}=-\lambda_{1} \ln \left(\lambda_{1}\right)-\lambda_{2} \ln \left(\lambda_{2}\right)-\lambda_{3} \ln \left(\lambda_{3}\right)
$$

Furthermore, the three eigenvalues may be normalized by their $\operatorname{sum} \Sigma_{\lambda}$ and the eigenentropy $E_{\lambda}$ can be computed by the Shannon entropy (Shannon, 1948) according to:

$$
\begin{gathered}
E_{\lambda}=-e_{1} \ln \left(e_{1}\right)-e_{2} \ln \left(e_{2}\right)-e_{3} \ln \left(e_{3}\right) \\
e_{i}=\frac{\lambda_{i}}{\Sigma_{\lambda}}, i \in\{1,2,3\} .
\end{gathered}
$$

The eigenentropy provides a measure of the 3D point's disorder within the covariance ellipsoid.

Variance and covariance are measures of the "spread" of a set of points around their center of mass (mean). In other words, the variance is the measure of the deviation from the mean for points in one dimension (e.g. heights) and covariance is a measure of how much each of the dimensions vary from the mean with respect to each other. The exact value of the covariance is not as important as its sign. A positive value shows that both dimensions increase or decrease together. A negative value shows that while one increases the other one decreases, or vice versa. If the value for the covariance is zero, the two dimensions are independent of each other.

The 3D covariance matrix, also known as 3D structure tensor is given by formula (13) and it is derived from the $3 \mathrm{D}$ coordinates of a given point $X=X_{0}$ and its $k$ nearest neighbors $(\mathrm{knn}) X_{i} \quad(i=$ $1 \ldots k)$ :

$$
S_{3 D}=\frac{1}{k+1} \sum_{i=0}^{k}\left(X_{i}-\bar{X}\right)\left(X_{i}-\bar{X}\right)^{T}
$$

where $\bar{X}=$ geometric center (Weinmann, 2016) in formula (14).

$$
\bar{X}=\frac{1}{k+1} \sum_{i=0}^{k} X_{i}
$$

The eigenvalues $\lambda_{1}, \lambda_{2}$, and $\lambda_{3}$ indicate the extent of a 3D ellipsoid along its three principal axes and it is displayed in Figure 5 (Lin et al., 2014).

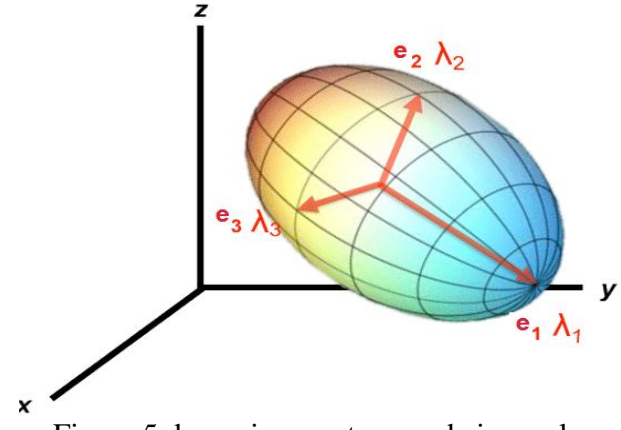

Figure 5. knn, eigenvectors, and eigenvalues

(Eigenvectors, 2020)

\subsection{Feature Selection}

In this step, the most suitable features are selected, so that the classification process does not consider the irrelevant and redundant ones. The accuracy of a classifier depends on the number of training samples. The curse of dimensionality or Hughes phenomenon shows this dependence in Figure 6. (Hughes, 1968). The same happens for the number of eigenfeatures computed for the points. In the literature are known two types of filter-based methods for feature selection. The univariate methods evaluate feature-class relations by the correlation coefficient. The multivariate methods evaluate both feature-class and feature-feature relations (Hall, 1999).

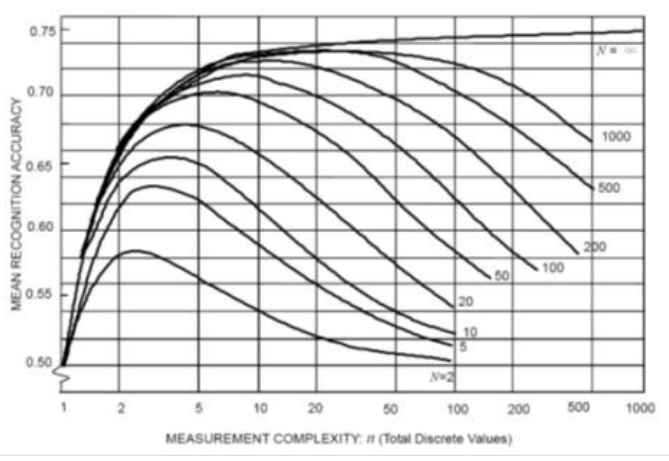

Figure 6. Hughes phenomenon (Chen et al., 2011)

\subsection{Classification}

The supervised classification methods can be categorized in individual classification where each $3 \mathrm{D}$ point is classified by only exploiting the respective feature vector and contextual classification where each 3D point is classified by exploiting the respective feature vector as well as contextual information derived from the feature vectors and class labels of neighboring 3D point (Weinmann et al., 2015).

The most known individual classification approaches are Maximum Likelihood classifiers, Support Vector Machines, and Random Forests.

Random Forest classifier (Breiman, 2001) uses an ensemble of classification trees, gets a prediction from each tree, and selects the best solution by a voting scheme.

$$
\lambda_{1} \geq \lambda_{2} \geq \lambda_{3}
$$


In (Li et al., 2019) the most well-known contextual classification approaches are explained and a novel approach is displayed. In the presented approach the following aspects are taken into account: each point can collect sufficient labelrelevant neighborhood information to verify its label based on an optimal graph and the input label probability set is improved by probabilistic label relaxation to be more consistent with the spatial context.

Markov Random Fields (Anguelov et al., 2005) and Conditional Random Fields (Niemeyer et al., 2012) are the two commonly used models for the contextual classification of 3D points. MRFs are generative models and encourage that the local neighborhood shares the same label. With CRFs the label dependencies between adjacency nodes are inferred by incorporating neighborhood spatial interactions among labels and can directly estimate conditional probabilities from the given features. Therefore the CRFs have been more widely used for point-cloud contextual classification.

\section{METHODOLOGY}

\subsection{Region Growing}

To answer differently to the question "How to recover an optimal value for $k$ ? " then we described in Section 2.2., we used the region growing algorithm to determine the optimal knn for each 3D point.

In the process of region growing the point normals and their residuals, under the user-specified parameters are used to group points belonging to the same smooth surfaces (Rabbani et al., 2006).

The algorithm sorts the points by their curvature value, to begin the region growing from the seed, which is the point that has the minimum curvature value. For every seed, the algorithm identifies the neighbors and computes the angles between the normal of the neighbor and the normal of the seed. If the angle for a specified point is less than a threshold value, then that point is added to the current smooth surface. This step is continued until the last neighbor is tested for his curvature value. If the seeds dataset becomes empty, it means that the algorithm has grown the region and the process is repeated from the beginning (PCL, 2019).

\subsection{Classification}

The classification process is composed of training, classification, and accuracy assessment.

For the classification process, we have used a machine learning algorithm, called 1 versus 1 classifier, who got good results in other studies, like (Grilli et al., 2019). This is a tool for turning binary classifiers into a multiclass classifier. It does this by training the binary classifiers in a one versus one fashion. So, for $N$ possible classes, the classifier trains $N^{*}(N-1) / 2$ binary classifiers, and classifies each point according to a voting scheme (King, 2009). For the classification accuracy, we compute the precision, recall and $\mathrm{F}_{1}$ score for every class, based on the values from the confusion matrix. In this matrix, the true positive (TP), true negative (TN), false positive (FP), and false negative (FN) values are computed based on the comparison between the predicted and the reference labels.

$$
\text { Precision }=\text { Correctness } s_{i}=\frac{T P_{i}}{T P_{i}+F P_{i}}
$$

$$
\begin{aligned}
& \text { Recall }=\text { Completness }_{i}=\frac{T P_{i}}{T P_{i}+F N_{i}} \\
& F_{1} \text { Score }_{i}=2 \frac{\text { Completness }_{i} * \text { Correctness }_{i}}{\text { Completness }_{i}+\text { Correctness }_{i}}
\end{aligned}
$$

\section{EXPERIMETS AND RESULTS}

\subsection{Dataset used}

For our experiments, we used a Romanian Dataset from LAKI II Project. The major activities of the project comprise the production of a detailed terrain model by LIDAR scanning, orthophotos, and 3D vector maps for $50.000 \mathrm{~km}^{2}$ of Romania's surface area. For LiDAR, the flight was conducted in 2017, with the RIEGL Q780 scanner. The average point cloud density was 8 points $/ \mathrm{m}^{2}$ and the vertical accuracy is $30 \mathrm{~cm}$.

For this work, we used a small dataset from the city of Marghita, Bihor County (Figure 7). The number of points in the dataset used was approximately 1.000 .000 and the density varied between 1 and 28 points $/ \mathrm{m}^{2}$ (Figure 8 ).

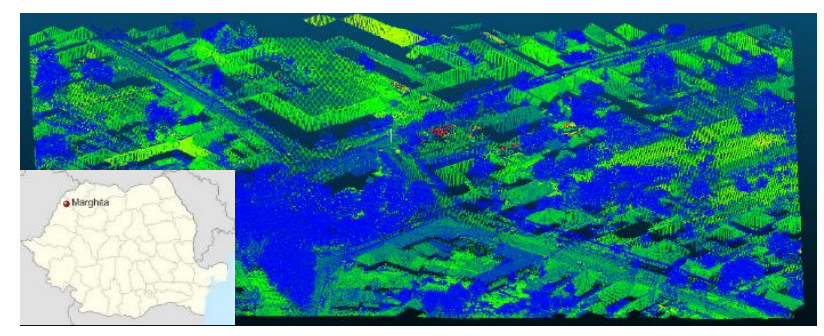

Figure 7. Employed dataset for the methodology evaluation

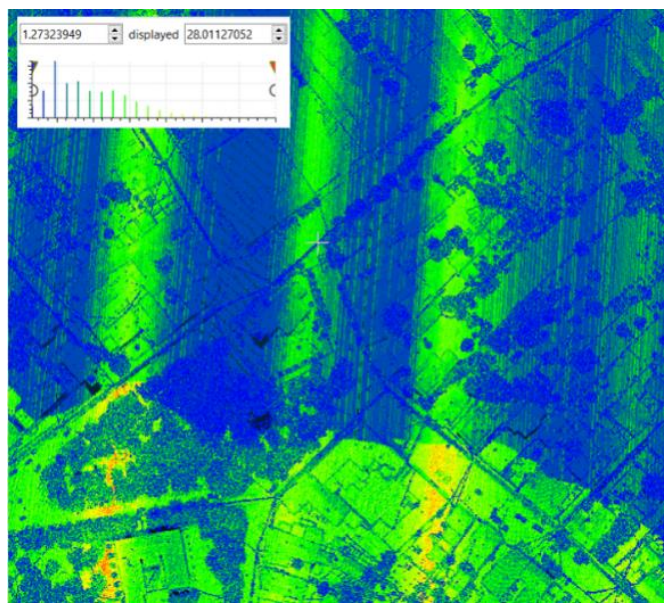

Figure 8. Point density in the dataset

In the initial point cloud, every point had the following attributes: $\mathrm{x}, \mathrm{y}, \mathrm{z}$ coordinates, intensity and return number. We manually labeled datasets for training (Figure 9) and for testing (Figure 10) using the tools from Cloud Compare software, in the following classes: 0 - ground, 1 - vegetation, 2 - roofs and 3 - others.

In Figure 9 and Figure 10, the classes are displayed with the following colors: ground - color blue, vegetation - color green, roofs - color yellow and others - color red. The class others is composed of points that represent cars, cable lines, poles, and other elements that couldn't be well distinguished in the scene. The number of points is displayed in Table 1 . 


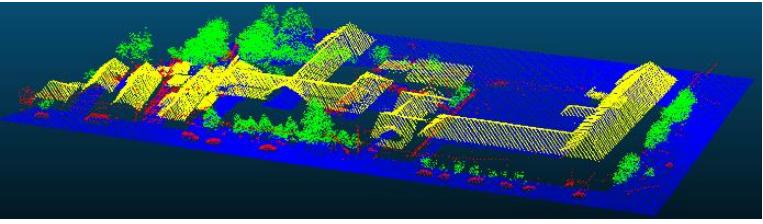

Figure 9. The training dataset (88 623 points)

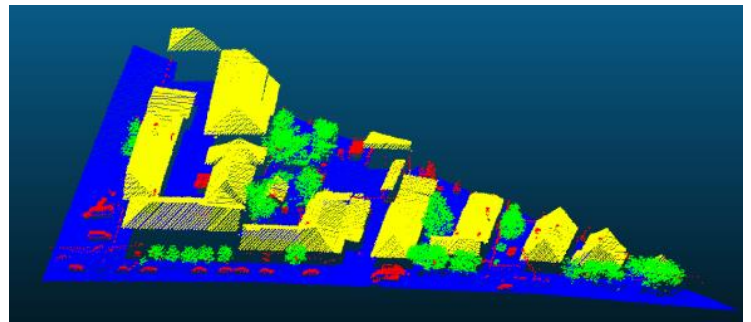

Figure 10 . The testing/reference dataset (85 470 points)

\begin{tabular}{|c|c|c|}
\hline \multirow{2}{*}{ Classes } & \multicolumn{2}{|c|}{ Number of points/Class } \\
\cline { 2 - 3 } & Training dataset & Testing dataset \\
\hline Ground & 49053 & 41804 \\
\hline Vegetation & 13770 & 11515 \\
\hline Roofs & 22397 & 29095 \\
\hline Others & 3403 & 3056 \\
\hline
\end{tabular}

Table 1. Number of points per class

\subsection{Experiments}

The first step in the point cloud processing was to remove the noise. In our experiment, we have used the Filter Noise instrument from Cloud Compare software and additional, we manually removed the isolated points in the dataset.

The idea used to determine the knn was to apply the region growing algorithm to determine the knn for each point. To select the best parameters for the region growing, we developed a $\mathrm{C}++$ script and made tests on three areas (Figure 11).

Test area 1 was made of 4712 points and comprised points on a tall building (roof and some points on the facade), on cars, and on the ground (Figure 11a). Test area 2 had 5840 points and comprised points on a roof, cars, and bushes (Figure 11b). The third test area was made of 13508 points and had points on cars, trees, poles, and power lines (Figure 11c).

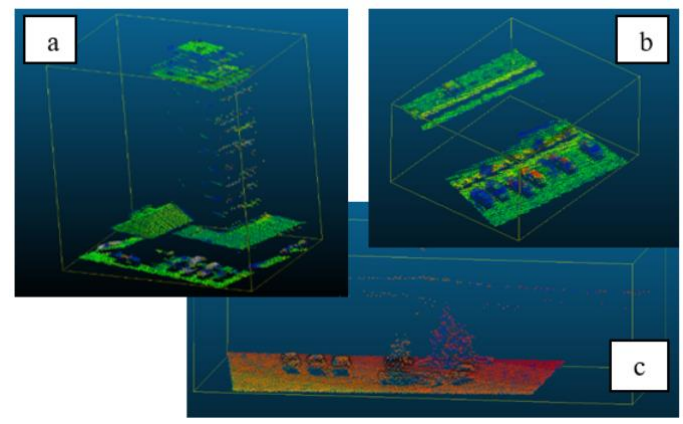

Figure 11. Tests areas for region growing (a. Test area 1, b. Test area 2, c. Test area 3)
In the region growing algorithm, every point becomes a seed and the cluster increases with the points surrounding the seed, based on the values of the parameters. So, for each point, in the script were generated two point clouds, as follows:

- one with the neighbourhood based on knn (a given value) - Figure 12a;

- one with the grown points based on the parameters (these points will be used as the neighbourhoods' seeds in the following steps) - Figure $12 \mathrm{~b}$.
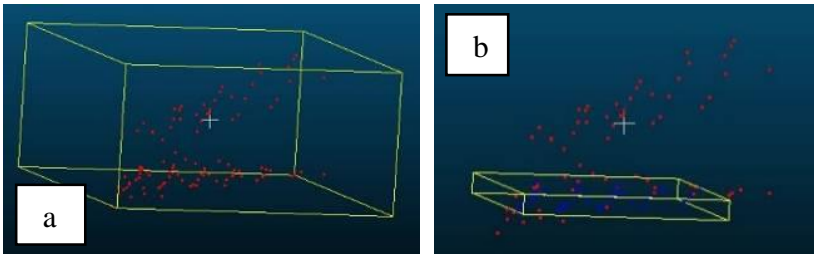

Figure 12. Example of point's selection using the region growing algorithm (a. neighbourhood based on knn, b. grown points with blue colour)

After a lot of tests on the areas described above, we chose the best parameters for region growing (Table 2).

\begin{tabular}{|c|c|}
\hline Parameters & $\begin{array}{c}\text { Optimal } \\
\text { values }\end{array}$ \\
\hline No. of neighbors for computation of the normals & 15 \\
\hline K For Kd Tree & 50 \\
\hline Minimum cluster size & 20 \\
\hline Maximum cluster size & 100 \\
\hline Number of neighbors & 15 \\
\hline Curvature threshold & 0.3 \\
\hline Smoothness threshold & $12.5 / 180^{*} \pi$ \\
\hline
\end{tabular}

Table 2. Region growing parameters

In the following, we computed the eigenfeatures using two approaches:

- $\quad$ based on a fixed $\mathrm{knn}(\mathrm{k}=50)$;

- based on a dynamic knn selected by region growing.

In Figure 13, we observed that the cable lines and the corners of the houses were better distinguished when we applied the optimization algorithm - dynamic knn.

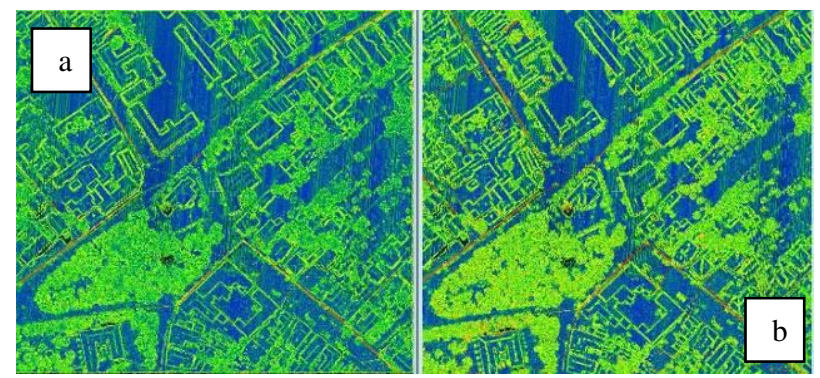

Figure 13. Point cloud displayed by linearity (a. fixed knn, b. dynamic knn)

In another $\mathrm{C}++$ script, we computed the following attributes for every point:

- $\quad \mathrm{N}_{\mathrm{x}}, \mathrm{N}_{\mathrm{y}}, \mathrm{N}_{\mathrm{z}}$ normals;

- curvature;

- $\quad \lambda_{1}, \lambda_{2}, \lambda_{3}$ eigenvalues; 
- $\quad$ eigenfeatures - 7;

- $\quad$ eigenvectors.

Based on the initial and computed attributes, we classified the point cloud using different combinations of the features. After some tests, we concluded that the accuracy is higher if we use the normalized values for the height and intensity. So, we introduced in the script these new formulas and tested other combinations of the features.

The last step in the workflow was the point cloud classification. We ran a script in $\mathrm{C}++$, starting from the Dlib library (DLIB, 2019) and performed the classification using One vs One classifier, based on the following features: normalized height, linearity, planarity, surface variation, sphericity, omnivariance, anisotropy, and normalized intensity.

For 4 classes (ground, vegetation, roofs, others), One vs One gives 6 binary classifiers. After training the classifier, we performed the classification for our dataset, based on all the 6 binary classifiers.

After analysing the values from the confusion matrix (Table 3), we concluded that the best results were obtained for the classes' vegetation and roofs.

\begin{tabular}{|c|c|c|c|c|}
\hline $\begin{array}{c}\text { Confusion } \\
\text { matrix }\end{array}$ & ground & vegetation & roofs & others \\
\hline ground & 443252 & 343771 & 115575 & 9023 \\
\hline vegetation & 214 & 12474 & 854 & 331 \\
\hline roofs & 1250 & 312 & 20710 & 165 \\
\hline others & 1288 & 13752 & 17729 & 1218 \\
\hline
\end{tabular}

Table 3. Confusion matrix (fixed knn)

Over $90 \%$ of the vegetation points were classified as true positives. The worst-case was obtained for the others' class, where the true positives represent only $4 \%$. A subset of the classification result is displayed in Figure 14.

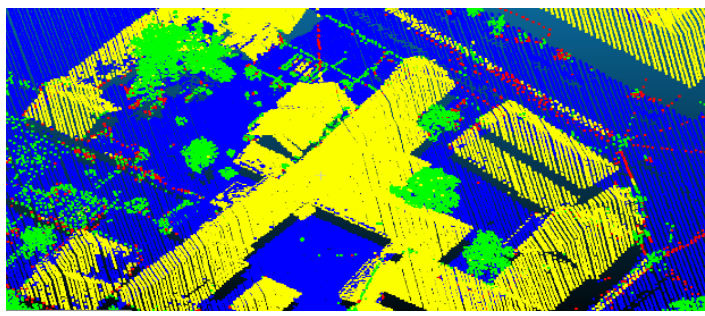

Figure 14. Classified point cloud with fixed knn

The results of the dynamic approach are displayed in Table 4 and Figure 15.

\begin{tabular}{|c|c|c|c|c|}
\hline $\begin{array}{c}\text { Confusion } \\
\text { matrix }\end{array}$ & ground & vegetation & roofs & others \\
\hline ground & 446390 & 327655 & 128877 & 8699 \\
\hline vegetation & 270 & 12277 & 1056 & 270 \\
\hline roofs & 1354 & 318 & 20683 & 82 \\
\hline others & 1299 & 12496 & 19308 & 884 \\
\hline
\end{tabular}

Table 4. Confusion matrix (dynamic knn)

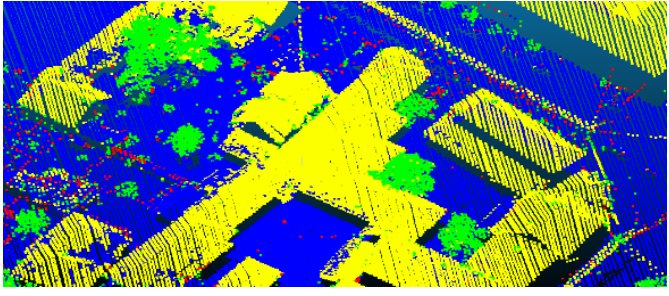

Figure 15. Classified point cloud with dynamic knn

The comparison between the accuracy indicators is displayed in Table 5. After analyzing the results we can state that the most complete class is the ground, and the most correct one is the roofs.

\begin{tabular}{|c|c|c|c|c|c|c|}
\hline \multirow{2}{*}{ Class } & \multicolumn{2}{|c|}{ Precision } & \multicolumn{2}{c|}{ Recall } & \multicolumn{2}{c|}{ F $_{1}$ Score } \\
\cline { 2 - 7 } & $\begin{array}{c}\text { fixed } \\
\text { knn }\end{array}$ & $\begin{array}{c}\text { dynam. } \\
\text { knn }\end{array}$ & $\begin{array}{c}\text { fixed } \\
\text { knn }\end{array}$ & $\begin{array}{c}\text { dynam } \\
\text { knn }\end{array}$ & $\begin{array}{c}\text { fixed } \\
\text { knn }\end{array}$ & $\begin{array}{c}\text { dynam. } \\
\text { knn }\end{array}$ \\
\hline ground & 48.6 & $49 \nearrow$ & 99.4 & $99.3 \searrow$ & 65.3 & $65.6 \nearrow$ \\
\hline vegeta. & 90 & $88.5 \downarrow$ & 3.3 & $3.5 \nearrow$ & 6.5 & $6.7 \nearrow$ \\
\hline roofs & 92.3 & $92.2 \downarrow$ & 13.4 & $12.2 \downarrow$ & 23.3 & $21.5 \downarrow$ \\
\hline others & 3.5 & $2.6 \downarrow$ & 11.3 & $8.9 \downarrow$ & 5.4 & $4.0 \downarrow$ \\
\hline
\end{tabular}

Table 5. Comparison between accuracy indicators

The slight improvement gained with the dynamic approach of computing the eigenfeatures is due in the most part to the noise in the dataset. These noisy points are very close to the ground, only 50 centimetres above it. So, their removal is a very difficult task.

Because of the multitude of distinct elements that composed the others class, its classification is difficult to be achieved with a large percentage.

\section{CONCLUSIONS}

The paper presented some investigations on methods for aerial point cloud classification. In every classification result, the quality of the input data is important. If the dataset has variable densities in the point cloud and a lot of noise, the results are not satisfying.

The selection of suitable features used for the classification is very important and in our future works, we will apply filterbased methods for feature selection.

For different datasets, the parameters used in the region growing algorithm need to be changed. So, these aspects must be studied more in the future.

From our point of view, some of the problems in the classification came from the others class, because the points from the training and the reference datasets were labelled manually, by a single person. The crowdsourcing is a wellknown practice of obtaining better results and we will use it for our future studies.

To extract the benefits of this new approach, in the future, we should test it with more datasets featuring different resolutions. 


\section{ACKNOWLEDGEMENTS}

The authors thank the National Centre of Cartography and the National Agency of Cadastre and Land Registration for providing the dataset used. The presented work was partly supported by the VOLTA project (Grant Agreement no. 734687 - VOLTA - H2020-MSCA-RISE-2016) and partly executed during a secondment of the first author in FBK Trento, Italy.

\section{References:}

Ben-Joseph, E., Ishii, H., Underkoffler, J., Piper, B., Yeung, L., 2001. Urban simulation and the luminous planning table: Bridging the gap between the digital and the tangible. Journal of Planning Education and Research, 21 (2), pp. 196-203.

Breiman, L., 2001. Random forests. Machine learning, 45(1), pp. 5-32.

Demantké, J., Mallet, C., David, N., Vallet, B., 2011. Dimensionality based scale selection in 3D lidar point clouds. The International Archives of the Photogrammetry, Remote Sensing and Spatial Information Sciences, XXXVIII-5/W12, pp. 97-102.

Filin, S., Pfeifer, N., 2005. Neighborhood systems for airborne laser data. Photogrammetric Engineering and Remote Sensing, 71(6), pp. 743-755.

Grilli, E., Özdemir, E., Remondino, F., 2019. Application of machine and deep learning strategies for the classification of heritage point clouds. The International Archives of the Photogrammetry, Remote Sensing and Spatial Information Sciences, XLII-4/W18.

Gross, H., Jutzi, B., Thoennessen, U., 2007. Segmentation of tree regions using data of a fullwavform laser. The International Archives of the Photogrammetry, Remote Sensing and Spatial Information Sciences, XXXVI-3/W49A:57-62.

Hughes, G. F., 1968. On the Mean Accuracy of Statistical Pattern Recognizers. IEEE Transactions on Information Theory, IT-14, pp. 55-63.

King, D. E., 2009. Dlib-ml: A Machine Learning Toolkit. Journal of Machine Learning Research, 10, pp. 1755-1758.

Lee, I., Schenk, T., 2002. Perceptual organization of 3D surface points. The International Archives of the Photogrammetry, Remote Sensing and Spatial Information Sciences, XXXIV (3A), pp. 193-198.

Li, N., Liu, C., Pfeifer, N., 2019. Improving LiDAR classification accuracy by contextual label smoothing in postprocessing. ISPRS Journal of Photogrammetry and Remote Sensing, 148, pp. 13-31.

Lin, C.-H., Chen, J.-Y., Su, P.-L., Chen, C.-H., 2014. Eigenfeature analysis of weighted covariance matrices for LiDAR point cloud classification. ISPRS Journal of Photogrammetry and Remote Sensing, 94, pp. 70-79.
Niemeyer, J., Rottensteiner, F., Soergel, U., 2012. Conditional random fields for LiDAR point cloud classification in complex urban areas. ISPRS Annals of the Photogrammetry, Remote Sensing and Spatial Information Sciences, 3, pp. 263-268.

Rabbani, T., Heuvel, F.A., Vosselman, G., 2006. Segmentation of point clouds using smoothness constraint. International Archives of Photogrammetry, Remote Sensing and Spatial Information Sciences, 36, pp. 248-253.

Shannon, C. E., 1948. A mathematical theory of communication. The Bell System Technical Journal, 27(3), pp. $379-423$.

Serna, A., Marcotegui, B., 2014. Detection, segmentation and classification of 3D urban objects using mathematical morphology and supervised learning. ISPRS Journal of Photogrammetry and Remote Sensing, 93, DOI: 10.1016/j.isprsjprs.2014.03.015.

Weinmann, M., Jutzi, B., Mallet, C., 2014. Semantic 3D scene interpretation: a framework combining optimal neighborhood size selection with relevant features. ISPRS Annals of the Photogrammetry, Remote Sensing and Spatial Information Sciences, II-3, pp. 181-188.

Weinmann, M., Schmidt, A., Mallet, C., Hinz, S., Rottensteiner, F. et al., 2015. Contextual classification of point cloud data by exploiting individual 3D neigbourhoods. ISPRS Annals of the Photogrammetry, Remote Sensing and Spatial Information Sciences II-3, pp. 271-278.

Wen, C., Yang, L., Li, X., Peng, L., Chi, T., 2020.Directionally constrained fully convolutional neural network for airborne LiDAR point cloud classification, ISPRS Journal of Photogrammetry and Remote Sensing, 162, pp. 50-62.

Zhao, K., Popescu, S., Nelson, R., 2009. Lidar remote sensing of forest biomass: A scale-invariant estimation approach using airborne lasers. Remote Sensing of Environment, 113, pp. 182196.

Jolliffe, I.T., 2002: Principal Component Analysis. Springer Series in Statistics, New York, pp. 10-28.

Weinmann, M., 2016: Reconstruction and Analysis of 3D Scenes. Springer, Karlsruhe, pp. 39-51, 152-164.

Anguelov, D., Taskarf, B., Chatalbashev, V., Koller, D., Gupta, D., Heitz, G., Ng, A., 2005. Discriminative learning of Markov Random Fields for segmentation of 3D scan data. IEEE Computer Society Conference on Computer Vision and Pattern Recognition, Vol. 2, pp. 169-176.

Gaurav, S. G., 2017. Light Detection and Ranging (LiDAR): Technologies and Global Markets. BCC Research report.

Hall, M. A., 1999. Doctoral Thesis. Correlation-based Feature Selection for Machine Learning, University of Waikato, Hamilton, New Zealand, pp. 25-51.

Weinmann, M., 2013. Visual Features-From Early Concepts to Modern Computer Vision. In: Advanced Topics in Computer Vision, Springer, London, pp. 1-34. 
Chen., I-L., Kuo, B.-C., Li, C.-H., Hung, C.-C., Combining ensemble technique of support vector machines with the optimal kernel method for high dimensional data classification. Powerpoint Presentation, July 28, 2011.

DLIB, 2019: https://dlib.net (view at 21 May 2020).

Eigenvectors, 2020: http://mriquestions.com/dti-tensorimaging.html (view at 5 September 2020).

PCL, 2019:

https://pcl.readthedocs.io/projects/tutorials/en/latest/region_gro wing_segmentation.html?highlight=region (view at 07 June 2020).

VOLTA, 2017: http://volta.fbk.eu (view at 29 September 2020). 